\title{
On a theorem of Erdős and Fuchs
}

\author{
by \\ GÁBOR Horváth (Budapest)
}

Let $k \geq 2$ be a fixed integer, let $A^{(j)}=\left\{a_{1}^{(j)}, a_{2}^{(j)}, \ldots\right\}(j=1, \ldots, k)$ be nondecreasing infinite sequences of nonnegative integers, and let

$r_{k}(n)=\left|\left\{\left(i_{1}, \ldots, i_{k}\right): a_{i_{1}}^{(1)}+a_{i_{2}}^{(2)}+\ldots+a_{i_{k}}^{(k)} \leq n, a_{i_{j}}^{(j)} \in A^{(j)}(j=1, \ldots, k)\right\}\right|$, and $c>0$.

Erdős and Fuchs [1] showed that if $k=2$ and $A^{(1)} \equiv A^{(2)}$, then

$$
r_{2}(n)=c n+o\left(n^{1 / 4}(\log n)^{-1 / 2}\right)
$$

cannot hold.

Sárközy [3] extended this theorem to two sequences which are "near" in a certain sense. He proved that if

$$
a_{i}^{(2)}-a_{i}^{(1)}=o\left(\left(a_{i}^{(1)}\right)^{1 / 2}\left(\log a_{i}^{(1)}\right)^{-1}\right),
$$

then (1) cannot hold. (A simple example shows that a condition of type (2) is necessary: Let $A^{(j)}=\left\{\sum_{l} \varepsilon_{l} 2^{l k+j}: \varepsilon_{l}=0\right.$ or 1$\}$ for $j=1, \ldots, k$. Then $r_{k}(n)=n+1$, thus $r_{k}(n)-n=O(1)$.)

In [2] I extended this result to the case $k>2$ and, among other things, I showed that if we assume

$$
a_{i}^{(j)}-a_{i}^{(l)}=o\left(\left(\min \left(a_{i}^{(j)}, a_{i}^{(l)}\right)\right)^{1 / 2}\left(\log \min \left(a_{i}^{(j)}, a_{i}^{(l)}\right)\right)^{-1-1 /(k-1)}\right)
$$

for all $1 \leq j<l \leq k$, then

$$
r_{k}(n)=c n+o\left(n^{1 / 4}(\log n)^{-1 / 2-3 /(2(k-1))}\right)
$$

cannot hold. In this paper I will show that, at the price of replacing the error term in (4) by a slightly weaker one, condition (3) can be replaced by a much weaker assumption. Namely, perhaps somewhat unexpectedly, it suffices to assume that two of the given sequences $A^{(j)}$ are "near":

2000 Mathematics Subject Classification: Primary 11B34. 
Theorem. If $k \geq 2, a_{i}^{(1)}-a_{i}^{(2)}=o\left(\left(a_{i}^{(1)}\right)^{1 / 2}\left(\log a_{i}^{(1)}\right)^{-k / 2}\right)$ and

$$
\sum_{a_{i}^{(j)} \leq N} 1 \ll \sum_{a_{i}^{(1)} \leq N} 1 \ll \sum_{a_{i}^{(j)} \leq N} 1 \quad \text { for } j=3, \ldots, k,
$$

then

$$
r_{k}(n)=c n+o\left(n^{1 / 4}(\log n)^{1-3 k / 4}\right)
$$

cannot hold.

Proof. Suppose that (5) holds. Let $v(n)=r_{k}(n)-c n$ and $F_{j}(z)=$ $\sum_{i=1}^{\infty} z^{a_{i}^{(j)}}(j=1, \ldots, k)$. Then for $|z|<1$,

$$
\begin{aligned}
\frac{1}{1-z} F_{1}(z) \ldots F_{k}(z) & =\sum_{n=0}^{\infty} r_{k}(n) z^{n}=c \sum_{n=0}^{\infty} n z^{n}+\sum_{n=0}^{\infty} v(n) z^{n} \\
& =c \frac{z}{(1-z)^{2}}+\sum_{n=0}^{\infty} v(n) z^{n}
\end{aligned}
$$

hence

$$
F_{1}(z) \ldots F_{k}(z)=\frac{c z}{1-z}+(1-z) \sum_{n=0}^{\infty} v(n) z^{n} .
$$

Let $\varepsilon$ be a fixed small positive number, $N$ a large positive integer, $m(n)=$ $\left[\varepsilon n^{1 / 2}(\log n)^{-k / 2}\right], m=m(N), z=r e(\alpha)$, where $r=1-1 / N$ and $e(\alpha)=$ $e^{2 \pi i \alpha}($ for real $\alpha)$. Let

$$
\begin{aligned}
J & =\int_{0}^{1}\left|F_{1}(z) \ldots F_{k}(z)\right|\left|\frac{1-z^{m}}{1-z}\right|^{2} d \alpha, \\
J_{1} & =c \int_{0}^{1}|1-z|^{-1}\left|\frac{1-z^{m}}{1-z}\right|^{2} d \alpha \\
J_{2} & =\int_{0}^{1}\left|(1-z) \sum_{n=0}^{\infty} v(n) z^{n}\right|\left|\frac{1-z^{m}}{1-z}\right|^{2} d \alpha .
\end{aligned}
$$

Then, by (6),

$$
J \leq J_{1}+J_{2}
$$

We first estimate $J$. By (7),

$$
\begin{aligned}
J & \geq\left.\left|\int_{0}^{1} F_{1}(z) \overline{F_{2}(z)} F_{3}(z) \ldots F_{k}(z)\right| \frac{1-z^{m}}{1-z}\right|^{2} d \alpha \mid \\
& =\left|\int_{0}^{1}\left(F_{1}(z) \overline{F_{2}(z)} \sum_{t=0}^{m-1} r^{t} e(-t \alpha)\right)\left(F_{3}(z) \ldots F_{k}(z) \sum_{t=0}^{m-1} r^{t} e(t \alpha)\right) d \alpha\right| .
\end{aligned}
$$


Let

$$
\begin{gathered}
\sum_{b=-\infty}^{\infty} g_{b} e(b \alpha)=F_{1}(z) \overline{F_{2}(z)} \sum_{t=0}^{m-1} r^{t} e(-t \alpha) \\
\sum_{i=0}^{\infty} h_{i} e(i \alpha)=F_{3}(z) \ldots F_{k}(z) \sum_{t=0}^{m-1} r^{t} e(t \alpha)
\end{gathered}
$$

(so that all the coefficients $g_{b}, h_{i}$ are nonnegative). Then

$$
J \geq\left|\int_{0}^{1} \sum_{b=-\infty}^{\infty} g_{b} e(b \alpha) \sum_{i=0}^{\infty} h_{i} e(i \alpha) d \alpha\right|=\sum_{b+i=0} g_{b} h_{i} \geq \sum_{m / 4 \leq i \leq m / 2} g_{-i} h_{i} .
$$

If $m / 4 \leq i \leq m / 2$, then

$$
\begin{aligned}
h_{i} & =\sum_{\substack{a_{i_{3}}^{(3)}+\ldots+a_{i_{k}}^{(k)}+t=i \\
0 \leq t \leq m-1}} r^{a_{i_{3}}^{(3)}+\ldots+a_{i_{k}}^{(k)}+t} \\
& \geq r^{N} \sum_{\substack{a_{i_{3}}^{(3)}+\ldots+a_{i_{k}}^{(k)}+t=i \\
0 \leq t \leq m / 2}} 1 \gg \sum_{a_{i_{3}}^{(3)}+\ldots+a_{i_{k}}^{(k)} \leq m / 4} 1
\end{aligned}
$$

since $r^{N}=(1-1 / N)^{N} \rightarrow 1 / e$.

For $k>2$, since

$$
\sum_{a_{i_{j}}^{(j)} \leq m /(4(k-2))} 1 \gg \sum_{a_{i_{1}}^{(1)} \leq m /(4(k-2))} 1 \quad(j=3, \ldots, k),
$$

it follows that for $m / 4 \leq i \leq m / 2$,

$$
\begin{aligned}
h_{i} & \sum_{a_{i_{3}}^{(3)}+\ldots+a_{i_{k}}^{(k)} \leq m / 4} 1 \geq\left(\sum_{a_{i_{3}}^{(3)} \leq m /(4(k-2))} 1\right) \ldots\left(\sum_{a_{i_{k}}^{(k)} \leq m /(4(k-2))} 1\right) \\
& \left.\sum_{a_{i_{1}}^{(1)} \leq m /(4(k-2))} 1\right)^{k-2},
\end{aligned}
$$

and thus, by (9),

$$
\begin{aligned}
J & \sum_{m / 4 \leq i \leq m / 2} g_{-i}\left(\sum_{a_{i_{1}}^{(1)} \leq m /(4(k-2))} 1\right)^{k-2} \\
& =\left(\sum_{a_{i_{1}}^{(1)} \leq m /(4(k-2))} 1\right)^{k-2} \sum_{m / 4 \leq i \leq m / 2} g_{-i} .
\end{aligned}
$$

Since $m=m(N)=\left[\varepsilon N^{1 / 2}(\log N)^{-k / 2}\right]$ is eventually nondecreasing, and 
$a_{i_{1}}^{(1)}-a_{i_{1}}^{(2)}=o\left(\left(a_{i_{1}}^{(1)}\right)^{1 / 2}\left(\log a_{i_{1}}^{(1)}\right)^{-k / 2}\right)$, it follows that if $a_{i_{1}}^{(1)} \leq N$, then $\left|a_{i_{1}}^{(1)}-a_{i_{1}}^{(2)}\right| \leq m\left(a_{i_{1}}^{(1)}\right) / 4 \leq m(N) / 4=m / 4$ for all sufficiently large $a_{i_{1}}^{(1)}$. Hence, for all sufficiently large $N$, if $a_{i_{1}}^{(1)} \leq N$, then $\left|a_{i_{1}}^{(1)}-a_{i_{1}}^{(2)}\right| \leq m / 4$. If $a_{i_{1}}^{(1)} \leq N-m$, then $a_{i_{1}}^{(2)} \leq a_{i_{1}}^{(1)}+\left|a_{i_{1}}^{(2)}-a_{i_{1}}^{(1)}\right| \leq N-m+m / 4<N$ and

$$
\begin{aligned}
0 & =m / 4-m / 4 \leq i-\left|a_{i_{1}}^{(2)}-a_{i_{1}}^{(1)}\right| \leq i+a_{i_{1}}^{(1)}-a_{i_{1}}^{(2)} \leq i+\left|a_{i_{1}}^{(2)}-a_{i_{1}}^{(1)}\right| \\
& \leq m / 2+m / 4<m-1,
\end{aligned}
$$

thus

$$
\begin{aligned}
g_{-i} & =\sum_{\substack{a_{i_{1}}^{(1)}-a_{i_{2}}^{(2)}-t=-i \\
0 \leq t \leq m-1}} r^{a_{i_{1}}^{(1)}+a_{i_{2}}^{(2)}+t} \\
& \geq \sum_{\substack{a_{i_{1}}^{(1)}-a_{i_{1}}^{(2)}-t=-i \\
0 \leq t \leq m-1 \\
a_{i_{1}}^{(1)}, a_{i_{1}}^{(2)} \leq N}} r^{a_{i_{1}}^{(1)}+a_{i_{1}}^{(2)}+t} \geq r^{3 N} \sum_{a_{i_{1}}^{(1)} \leq N-m} 1 \gg \sum_{a_{i_{1}}^{(1)} \leq N-m} 1 .
\end{aligned}
$$

Hence, by (10) and (11),

$$
J \gg m\left(\sum_{a_{i_{1}}^{(1)} \leq m /(4(k-2))} 1\right)^{k-2} \sum_{a_{i_{1}}^{(1)} \leq N-m} 1 .
$$

Since $a_{i}^{(2)}-a_{i}^{(1)}=a_{i}^{(1)}\left(a_{i}^{(2)} / a_{i}^{(1)}-1\right)$ and $a_{i}^{(2)}-a_{i}^{(1)}=o\left(m\left(a_{i}^{(1)}\right)\right)$, so that $a_{i}^{(2)} / a_{i}^{(1)}=1+o\left(m\left(a_{i}^{(1)}\right) / a_{i}^{(1)}\right)=1+o(1)$, it follows that

$$
\begin{aligned}
& a_{i}^{(2)}-a_{i}^{(1)} \\
& =o\left(m\left(a_{i}^{(1)}\right)\right)=o\left(\left(a_{i}^{(1)}\right)^{1 / 2}\left(\log a_{i}^{(1)}\right)^{-k / 2}\right) \\
& =o\left(\left(a_{i}^{(2)}\right)^{1 / 2}\left(\log a_{i}^{(2)}\right)^{-k / 2}\right)\left(a_{i}^{(1)}\left(a_{i}^{(2)}\right)^{-1}\right)^{1 / 2}\left(\left(\log a_{i}^{(2)}\right)\left(\log a_{i}^{(1)}\right)^{-1}\right)^{k / 2} \\
& =o\left(\left(a_{i}^{(2)}\right)^{1 / 2}\left(\log a_{i}^{(2)}\right)^{-k / 2}\right)=o\left(m\left(a_{i}^{(2)}\right)\right) .
\end{aligned}
$$

As $m$ is eventually nondecreasing, it follows that if $a_{i}^{(2)} \leq N$, then $\left|a_{i}^{(1)}-a_{i}^{(2)}\right|$ $\leq m\left(a_{i}^{(2)}\right) / 4 \leq m(N) / 4=m / 4$ for all sufficiently large $a_{i}^{(2)}$. Hence, for all sufficiently large $N$, if $a_{i}^{(2)} \leq N$, then $\left|a_{i}^{(1)}-a_{i}^{(2)}\right| \leq m / 4$. Furthermore,

$$
\sum_{a_{i_{j}}^{(j)} \leq N-5 m / 4} 1 \ll \sum_{a_{i_{1}}^{(1)} \leq N-5 m / 4} 1 \text { for } j=3, \ldots, k,
$$


and $r_{k}(N) \sim c N$, thus

$$
\begin{aligned}
N & \ll r_{k}(N / 2) \leq r_{k}(N-[N / 2]) \leq r_{k}(N-5 m / 4) \\
& =\sum_{a_{i_{1}}^{(1)}+\ldots+a_{i_{k}}^{(k)} \leq N-5 m / 4} 1 \leq \prod_{j=1}^{k} \sum_{a_{i_{j}}^{(j)} \leq N-5 m / 4} 1 \\
& \ll\left(\prod_{\substack{j=1 \\
j \neq 2}}^{k} \sum_{a_{i_{1}}^{(1)} \leq N-5 m / 4} 1\right)\left(\sum_{a_{i_{2}}^{(1)} \leq N-m} 1\right) \leq\left(\sum_{a_{i_{1}}^{(1)} \leq N-m} 1\right)^{k},
\end{aligned}
$$

hence

$$
\sum_{a_{i_{1}}^{(1)} \leq N-m} 1 \gg N^{1 / k} .
$$

By a similar argument for $k>2$ and for all sufficiently large $N$, if $a_{i}^{(2)} \leq N$, then $\left|a_{i}^{(1)}-a_{i}^{(2)}\right| \leq m /(8(k-2))$. Thus

$$
\begin{aligned}
m & \ll r_{k}\left(\frac{m}{8(k-2)}\right) \leq \prod_{j=1}^{k} \sum_{a_{i_{j}}^{(j)} \leq m /(8(k-2))} 1 \\
& \ll\left(\prod_{\substack{j=1 \\
j \neq 2}}^{k} \sum_{a_{i_{1}}^{(1)} \leq m /(8(k-2))} 1\right)\left(\sum_{a_{i_{2}}^{(1)} \leq m /(4(k-2))} 1\right) \leq\left(\sum_{a_{i_{1}}^{(1)} \leq m /(4(k-2))} 1\right)^{k},
\end{aligned}
$$

hence

$$
\sum_{a_{i_{1}}^{(1)} \leq m /(4(k-2))} 1 \gg m^{1 / k} .
$$

By (12)-(14),

$$
J \gg m m^{(k-2) / k} N^{1 / k}=m^{2-2 / k} N^{1 / k} .
$$

We now estimate $J_{1}$ and $J_{2}$. Since

$$
\begin{aligned}
|1-z|^{2} & =(1-r \cos 2 \pi \alpha)^{2}+(r \sin 2 \pi \alpha)^{2}=(1-r)^{2}+2 r(1-\cos 2 \pi \alpha) \\
& =\frac{1}{N^{2}}+4 r \sin ^{2} \pi \alpha
\end{aligned}
$$

and

$$
|(2 / \pi) \pi \alpha| \leq|\sin \pi \alpha| \quad \text { for }|\alpha| \leq 1 / 2,
$$


it follows that $\max \left(1 / N^{2}, \alpha^{2}\right) \ll|1-z|^{2}$, thus $\max (1 / N, \alpha) \ll|1-z|$. Hence

$$
\begin{aligned}
J_{1} & =c \int_{0}^{1}|1-z|^{-1}\left|\frac{1-z^{m}}{1-z}\right|^{2} d \alpha \ll m^{2} \int_{0}^{1}|1-z|^{-1} d \alpha \\
& \ll m^{2}\left(\int_{0}^{1 / N}|1-z|^{-1} d \alpha+\int_{1 / N}^{1 / 2}|1-z|^{-1} d \alpha\right) \\
& \ll m^{2}\left(\frac{1}{N} N+\int_{1 / N}^{1 / 2} \frac{1}{\alpha} d \alpha\right) \leq m^{2}(1+\log N) \\
& \ll m^{2} \log N .
\end{aligned}
$$

By Cauchy's inequality and Parseval's formula,

$$
\begin{aligned}
J_{2} & =\int_{0}^{1}\left|(1-z) \sum_{n=0}^{\infty} v(n) z^{n}\right|\left|\frac{1-z^{m}}{1-z}\right|^{2} d \alpha \\
& \leq 2 \int_{0}^{1}\left|\sum_{n=0}^{\infty} v(n) z^{n}\right|\left|\frac{1-z^{m}}{1-z}\right| d \alpha \\
& \ll\left(\int_{0}^{1}\left|\sum_{n=0}^{\infty} v(n) z^{n}\right|^{2} d \alpha\right)^{1 / 2}\left(\int_{0}^{1}\left|\frac{1-z^{m}}{1-z}\right|^{2} d \alpha\right)^{1 / 2} \\
& \leq\left(\sum_{n=0}^{\infty}|v(n)|^{2} r^{2 n}\right)^{1 / 2} m^{1 / 2} .
\end{aligned}
$$

By our assumption, $v(n)=o\left(n^{1 / 4}(\log n)^{1-3 k / 4}\right)$, therefore for every $\eta>0$, there exists a natural number $K(\geq 2)$ such that for all $n \geq K$, $|v(n)| \leq \eta n^{1 / 4}(\log n)^{1-3 k / 4}$ and $n^{1 / 4}(\log n)^{1-3 k / 4}$ is nondecreasing. Then for all $N \geq K$,

$$
\begin{aligned}
\sum_{n=0}^{\infty}|v(n)|^{2} r^{2 n} \leq & \sum_{n=0}^{K-1}|v(n)|^{2}+\eta^{2} \sum_{n=K}^{\infty} n^{1 / 2}(\log n)^{2-3 k / 2} r^{2 n} \\
\leq & \sum_{n=0}^{K-1}|v(n)|^{2}+\eta^{2} N N^{1 / 2}(\log N)^{2-3 k / 2} \\
& +\eta^{2} \sum_{j=0}^{\infty} \sum_{n=2^{j} N+1}^{2^{j+1} N} n^{1 / 2}(\log n)^{2-3 k / 2} r^{n}
\end{aligned}
$$


Since

$$
\begin{aligned}
& \sum_{j=0}^{\infty} \sum_{n=2^{j} N+1}^{2^{j+1} N} n^{1 / 2}(\log n)^{2-3 k / 2} r^{n} \\
& \quad \leq \sum_{j=0}^{\infty} 2^{j} N\left(2^{j+1} N\right)^{1 / 2}\left(\log \left(2^{j+1} N\right)\right)^{2-3 k / 2} r^{2^{j} N} \\
& \quad \leq N^{3 / 2}(\log N)^{2-3 k / 2} \sum_{j=0}^{\infty} 2^{j+j / 2+1 / 2} e^{-2^{j}}=C_{0} N^{3 / 2}(\log N)^{2-3 k / 2}
\end{aligned}
$$

it follows that

$$
\begin{aligned}
\sum_{n=0}^{\infty}|v(n)|^{2} r^{2 n} & \leq \sum_{n=0}^{K-1}|v(n)|^{2}+\eta^{2} N^{3 / 2}(\log N)^{2-3 k / 2}\left(1+C_{0}\right) \\
& <\eta N^{3 / 2}(\log N)^{2-3 k / 2}
\end{aligned}
$$

for $\eta<\left(2\left(1+C_{0}\right)\right)^{-1}$ and for $N>N_{0}(\eta)$. Thus

$$
\sum_{n=0}^{\infty}|v(n)|^{2} r^{2 n}=o\left(N^{3 / 2}(\log N)^{2-3 k / 2}\right) .
$$

By (17) and (18),

$$
J_{2}=o\left(N^{3 / 4}(\log N)^{1-3 k / 4} m^{1 / 2}\right) .
$$

By (8), (15), (16), and (19),

$$
m^{2-2 / k} N^{1 / k} \ll m^{2} \log N+o\left(m^{1 / 2} N^{3 / 4}(\log N)^{1-3 k / 4}\right) .
$$

Since $m=\left[\varepsilon N^{1 / 2}(\log N)^{-k / 2}\right],(20)$ yields

$$
\left(\frac{\varepsilon}{2} N^{1 / 2}(\log N)^{-k / 2}\right)^{2-2 / k} N^{1 / k}
$$$$
\ll \varepsilon^{2} N(\log N)^{-k} \log N+o\left(\varepsilon^{1 / 2} N^{1 / 4}(\log N)^{-k / 4} N^{3 / 4}(\log N)^{1-3 k / 4}\right)
$$

for all sufficiently large $N$, hence $\varepsilon^{3 / 2-2 / k} \ll \varepsilon^{3 / 2}+o(1)$. Thus $\varepsilon^{-2 / k} \ll 1$; but this cannot hold for sufficiently small $\varepsilon$. This completes the proof of the theorem.

Acknowledgements. The author would like to thank Professors Imre Z. Ruzsa and András Sárközy for helpful suggestions.

\section{References}

[1] P. Erdős and W. H. J. Fuchs, On a problem of additive number theory, J. London Math. Soc. 31 (1956), 67-73.

[2] G. Horváth, On a generalization of theorem of Erdös and Fuchs, to appear. 
[3] A. Sárközy, On a theorem of Erdös and Fuchs, Acta Arith. 37 (1980), 333-338.

Department of Algebra and Number Theory

Eötvös Loránd University

Pázmány Péter sétány 1/c

H-1117, Budapest, Hungary

E-mail: horvathg@cs.elte.hu 